\title{
UNIQUENESS AND INTERPOLATION RESULTS FOR ENTIRE FUNCTIONS OF EXPONENTIAL TYPE ${ }^{1}$
}

\author{
BY JAMES D. CHILD \\ Communicated by R. Creighton Buck, November 30, 1972
}

1. Introduction. Let $K[\Omega]$ denote the collection of entire functions of exponential type whose Borel transforms are analytic on $\Omega^{c}$, the complement of $\Omega$ taken relative to the sphere. The domain $\Omega$ is assumed to be simply connected throughout the article. This note discusses a new approach for proving theorems about uniqueness classes of the form $K[\Omega]$ for sequences of linear functionals $\left\{L_{n}\right\}$, where

$$
L_{n}(f)=\frac{1}{2 \pi i} \int_{\Gamma} g_{n}(\lambda) F(\lambda) d \lambda \quad(n=0,1, \ldots) .
$$

Each $g_{n}(n=0,1, \ldots)$ is in $H(\Omega)$, the collection of all functions holomorphic on $\Omega, F$ denotes the Borel transform of $f$, and $\Gamma$ is a simple closed contour in $\Omega$ with $F$ holomorphic outside and on $\Gamma$. We shall also characterize sequences of complex numbers $\left\{b_{n}\right\}$ which can be interpolated by $K[\Omega]$ relative to $\left\{L_{n}\right\}$.

Our results on uniqueness and interpolation require the following setting. Place the topology of uniform convergence on compact subsets of $\Omega$ on $H(\Omega)$. Then the dual space of $H(\Omega)$ can be identified with the collection of functions holomorphic on $\Omega^{c}$ which vanish at $\infty$ [8]. This collection will be denoted by $H_{0}\left(\Omega^{c}\right)$. Note that $H_{0}\left(\Omega^{c}\right)$ is also the collection of Borel transforms of all functions in $K[\Omega]$. The bilinear form relating $H(\Omega)$ and $H_{0}\left(\Omega^{c}\right)$ is given by

$$
\langle\alpha, F\rangle=\frac{1}{2 \pi i} \int_{\Gamma} \alpha(\lambda) F(\lambda) d \lambda
$$

for each $\alpha$ in $H(\Omega)$ and each $F$ in $H_{0}\left(\Omega^{c}\right)$.

Let $\Omega_{\lambda}$ and $\Omega_{\zeta}$ be simply connected domains in the plane and let $T$ denote a continuous linear operator from $H\left(\Omega_{\lambda}\right)$ to $H\left(\Omega_{\zeta}\right)$. For $\left\{g_{n}\right\}$ with each $g_{n}$ in $H\left(\Omega_{\lambda}\right)$ define $\left\{L_{T_{n}}\right\}$ by

$$
L_{T_{n}}(f)=\frac{1}{2 \pi i} \int_{\Gamma}\left[T\left(g_{n}\right)\right](\zeta) F(\zeta) d \zeta \quad(n=0,1, \ldots)
$$

AMS (MOS) subject classifications (1970). Primary 30A66.

Key words and phrases. Entire functions of exponential type, dual space, conjugate operator, uniqueness results, interpolation results.

${ }^{1}$ These results are contained in the author's $1972 \mathrm{Ph} . \mathrm{D}$. dissertation at the University of Cincinnati, written under the supervision of Professor Richard F. DeMar. 
for each $f$ in $K\left[\Omega_{\zeta}\right]$. We shall look for special types of operators $T$. If $K\left[\Omega_{\lambda}\right]$ is a uniqueness class for $\left\{L_{n}\right\}$ implies that $K\left[\Omega_{\zeta}\right]$ is a uniqueness class for $\left\{L_{T_{n}}\right\}$, then $T$ is said to be uniqueness preserving. If $K\left[\Omega_{\lambda}\right]$ interpolates $\left\{b_{n}\right\}$ relative to $\left\{L_{n}\right\}$ implies that $K\left[\Omega_{\zeta}\right]$ interpolates $\left\{b_{n}\right\}$ relative to $\left\{L_{T_{n}}\right\}$, then $T$ is said to be interpolation preserving.

2. The results. Characterizations will first be obtained for uniqueness preserving operators, interpolation preserving operators, and operators which are both uniqueness preserving and interpolation preserving. These characterizations will then be applied to obtain uniqueness and interpolation results.

For each $F$ in $H_{0}\left(\Omega_{\zeta}^{c}\right)$ define $T^{\prime}(F)$ by $\left\langle\alpha, T^{\prime}(F)\right\rangle=\langle T(\alpha), F\rangle$ for all $\alpha$ in $H\left(\Omega_{\lambda}\right)$. The operator $T^{\prime}$ from $H_{0}\left(\Omega_{\xi}^{c}\right)$ to $H_{0}\left(\Omega_{\lambda}^{c}\right)$ thus defined is called the conjugate (dual) operator of $T$. Some characterizations of operators we shall find are in terms of the conjugate operator.

THEOREM 2.1. A necessary and sufficient condition for $T$ to be uniqueness preserving is that $T^{\prime}$ is one-to-one.

Proof. (Sufficiency) Suppose that $\left\langle T\left(g_{n}\right), F\right\rangle=0(n=0,1, \ldots)$, then $\left\langle g_{n}, T^{\prime}(F)\right\rangle=0(n=0,1, \ldots)$. If $K\left[\Omega_{\lambda}\right]$ is a uniqueness class for $\left\{L_{n}\right\}$, $T^{\prime}(F) \equiv 0$. By hypothesis $T^{\prime}$ is a one-to-one linear operator so that $F \equiv 0$. Hence, $f \equiv 0$.

(Necessity) Suppose there exists $F \not \equiv 0$ such that $T^{\prime}(F) \equiv 0$. Then, for each sequence $\left\{g_{n}\right\}$ from $H\left(\Omega_{\lambda}\right), 0=\left\langle g_{n}, T^{\prime}(F)\right\rangle=\left\langle T\left(g_{n}\right), F\right\rangle(n=0,1$, ...) and $F \not \equiv 0$ so that $K\left[\Omega_{\zeta}\right]$ cannot be a uniqueness class for $\left\{L_{T_{n}}\right\}$. But for $\left\{\boldsymbol{g}_{n}(\lambda)\right\}=\left\{\lambda^{n}\right\}, K\left[\Omega_{\lambda}\right]$ is a uniqueness class for the sequence of functionals generated by $\left\{\boldsymbol{g}_{\boldsymbol{n}}\right\}$.

REMARK 2.2. The conclusion of the proof of the necessity above may be restated as if there exists $\left\{g_{n}\right\}$ from $H\left(\Omega_{\lambda}\right)$ such that $K\left[\Omega_{\zeta}\right]$ is a uniqueness class for $\left\{L_{T_{n}}\right\}$, then $T$ is uniqueness preserving.

THEOREM 2.3. A necessary and sufficient condition for $T$ to be interpolation preserving is that $T^{\prime}$ maps onto $H_{0}\left(\Omega_{\lambda}^{c}\right)$.

Proof. (Sufficiency) Suppose that $\left\langle\mathrm{g}_{n}, G\right\rangle=b_{n}(n=0,1, \ldots)$. By hypothesis there exists $F$ in $H_{0}\left(\Omega_{\xi}^{c}\right)$ with $T^{\prime}(F)=G$. So, $b_{n}=\left\langle g_{n}, G\right\rangle$ $=\left\langle g_{n}, T^{\prime}(F)\right\rangle=\left\langle T\left(g_{n}\right), F\right\rangle(n=0,1, \ldots)$.

(Necessity) Set $g_{n}(\lambda)=\lambda^{n}(n=0,1, \ldots)$ and suppose that $T^{\prime}$ does not map onto $H_{0}\left(\Omega_{\lambda}^{c}\right)$. We assume that $T$ is interpolation preserving and show that a contradiction results. There exists $G$ in $H_{0}\left(\Omega_{\lambda}^{c}\right)$ such that $G$ is not in $T^{\prime}\left[H_{0}\left(\Omega_{\xi}^{c}\right)\right]$. Define $\left\{b_{n}\right\}$ by $b_{n}=\left\langle g_{n}, G\right\rangle(n=0,1, \ldots)$. By assumption there exists $F$ in $H_{0}\left(\Omega_{\lambda}^{c}\right)$ with $\left\langle T\left(g_{n}\right), F\right\rangle=b_{n}(n=0,1, \ldots)$. Thus for each $n=0,1, \ldots,\left\langle T\left(g_{n}\right), F\right\rangle=\left\langle g_{n}, T^{\prime}(F)\right\rangle=b_{n}=\left\langle g_{n}, G\right\rangle$. But $T^{\prime}(F) \neq G$ so that $K\left[\Omega_{\lambda}\right]$ is not a uniqueness class for the sequence of 
functionals corresponding to $\left\{\lambda^{n}\right\}$. This is a contradiction.

THEOREM 2.4. A necessary and sufficient condition for $T$ to be both uniqueness preserving and interpolation preserving is that $T$ is a homeomorphism.

Proof. (Sufficiency) It is known that $H\left(\Omega_{\lambda}\right)$ and $H\left(\Omega_{\zeta}\right)$ are Fréchet spaces, e.g. see Kothe [8]. Since $T$ is one-to-one and onto $H\left(\Omega_{\zeta}\right)$ implies that $T^{\prime}$ maps one-to-one and onto $H_{0}\left(\Omega_{\lambda}^{c}\right)[6]$, the result follows from Theorem 2.1 and Theorem 2.3.

(Necessity) By Theorem 2.1, $T$ uniqueness preserving implies that $T^{\prime}$ is one-to-one. By Theorem 2.3, $T$ interpolation preserving implies that $T^{\prime}$ maps onto $H_{0}\left(\Omega_{\lambda}^{c}\right)$. From known results $[6$, p. $521 \mathrm{ff}]$, this implies $T^{-1}$ is continuous.

3. Applications. Some of the more important known uniqueness and interpolation results deal with sequences of linear functionals $\left\{L_{n}\right\}$ which are generated by sequences of functions of the form $\left\{[W(\zeta)]^{n}\right\}$.

THEOREM 3.1. Let $W$ be analytic on $\Omega$. Then $K[\Omega]$ is a uniqueness class for $\left\{L_{n}\right\}$ generated by $\left\{[W(\zeta)]^{n}\right\}$ if and only if $W(\zeta)$ is univalent on $\Omega$.

Proof. (If) If $W$ is univalent on $\Omega$, then the operator $T$ defined on $H(W(\Omega))$ by $[T(\alpha)](\zeta)=\alpha(W(\zeta))$ is a linear homeomorphism onto $H(\Omega)$. But $K[W(\Omega)]$ is a uniqueness class for $\left\{L_{n}\right\}$ generated by $\left\{\lambda^{n}\right\}$ and the result follows from Theorem 2.4 .

(Only if) If $W$ is not univalent on $\Omega$, then $T^{\prime}$ is not one-to-one. For $\zeta_{1}, \zeta_{2}$ in $\Omega$ with $\zeta_{1} \neq \zeta_{2}$ and $W\left(\zeta_{1}\right)=W\left(\zeta_{2}\right)$, set $F(\zeta)=1 /\left(\zeta-\zeta_{1}\right)$ $-1 /\left(\zeta-\zeta_{2}\right)$. Then $T^{\prime}(F) \equiv 0$.

Gelfond proved Theorem 3.1 in 1938 [7], Buck using Mittag-Leffler summability gave a simpler proof of sufficiency for the case $W(0)=0$ and $W(\Omega)$ star-shaped with respect to the origin in 1948 [2], and DeMar [4] proved the sufficiency in 1963. Our approach is quite different.

TheOREM 3.2. Let $W$ be analytic and univalent on $\Omega$. Let $\left\{b_{n}\right\}$ be a sequence of complex numbers and define a function $B$ by

$$
B(\zeta)=\sum_{n=0}^{\infty} \frac{b_{n}}{\zeta^{n+1}}
$$

and its analytic continuation. Then $K[\Omega]$ interpolates $\left\{b_{n}\right\}$ relative to $\left\{L_{n}\right\}$ generated by $\left\{[W(\zeta)]^{n}\right\}$ if and only if $B$ is analytic on $[W(\Omega)]^{c}$.

PRoOF. The sequence $\left\{b_{n}\right\}$ can be interpolated by $K[W(\Omega)]$ relative to $\left\{f^{(n)}(0)\right\}$ if and only if there exists $g$ in $K[W(\Omega)]$ such that $\left\{g^{(n)}(0)\right\}=\left\{b_{n}\right\}$ if and only if the function $G$, the Borel transform of $g$, defined by 
$G(\zeta)=\sum_{n=0}^{\infty} b_{n} / \zeta^{n+1}$ and its analytic continuation is analytic on $[W(\Omega)]^{c}$. The operator $T$ defined in the proof of Theorem 3.1 is interpolation preserving. Thus, $\left\{b_{n}\right\}$ can be interpolated by $K[\Omega]$ if and only if $B=G$ is analytic on $[W(\Omega)]^{c}$.

In 1948 Buck [1] proved the necessity of Theorem 3.2 for the case $\Omega$ contains the origin and $W(0)=0$. DeMar [3] proved the sufficiency in 1961 for the case $W(0)=0$ and $\Omega$ is a convex set containing the origin. In 1963 DeMar [4] replaced the convexity requirement with the condition that $\Omega$ be simply connected.

The following two theorems lead to new uniqueness and interpolation results when the operators specified in the theorems are applied to the generating functions of sequences of linear functionals for which uniqueness and interpolation results are already known.

THEOREM 3.3. The operator $T$ defined on $H(\Omega)$ by $T(\alpha)=\alpha^{\prime}$ is uniqueness preserving but not interpolation preserving.

THEOREM 3.4. Let $\Omega$ contain the origin, then $T$ defined on $H(\Omega)$ by $[T(\alpha)](\zeta)=\alpha(\zeta)+\zeta \alpha^{\prime}(\zeta)$ is both uniqueness preserving and interpolation preserving.

\section{REFERENCES}

1. R. C. Buck, Integral valued entire functions, Duke Math. J. 15 (1948), 879-891. MR 10, 693.

2. Interpolation series, Trans. Amer. Math. Soc. 64 (1948), 283-298. MR 10, 693.

3. R. F. DeMar, Existence of interpolating functions of exponential types, Trans. Amer. Math. Soc. 105 (1962), 359-371. MR 25 \#5317.

4. - On a theorem concerning existence of interpolating functions, Trans. Amer. Math. Soc. 114 (1965), 23-29. MR 35 \# 1788.

5. - A uniqueness theorem for entire functions, Proc. Amer. Math. Soc. 16 (1965), 69-71. MR 30 \# 2145.

6. R. E. Edwards, Functional analysis, theory and application, Holt, Rinehart and Winston, New York, 1965. MR 36 \# 4308.

7. A. O. Gelfond, Interpolation et unicité des fonctions entières, Rec. Math. Moscow (N.S.) 4 (1938), 115-147.

8. G. Köthe, Topological linear spaces. I, Die Grundlehren der Math. Wissenschaften, Band 159, Springer-Verlag, New York, 1969. MR 40 \# 1750.

Department of Mathematics, University of Cincinnati, Cincinnati, Ohio 45221

Department of Mathematics, Wilson College, Chambersburg, Pennsylvania 17201 (current address) 